\title{
Successful Treatment of Growing Teratoma Syndrome of the Lung by Surgical Resection: A Case Report and Literature Review
}

\author{
KENSUKE TANAKA ${ }^{1}$, GOUJI TOYOKAWA ${ }^{1}$, TETSUZO TAGAWA ${ }^{1}$, KAYO IJICHI $^{2}$, \\ NAOKI HARATAKE ${ }^{1}$, FUMIHIKO HIRAI ${ }^{1}$, YOSHINAO ODA ${ }^{2}$ and YOSHIHIKO MAEHARA ${ }^{1}$ \\ ${ }^{1}$ Department of Surgery and Sciences, ${ }^{2}$ Department of Anatomic Pathology, \\ Graduate School of Medical Sciences, Kyushu University, Fukuoka, Japan
}

\begin{abstract}
Growing teratoma syndrome (GTS) of the lung is extremely rare, and there are very few reports on this condition. This is a case report of GTS of the lung that was successfully treated by resection. A 19-year-old man, who had been diagnosed with a testicular tumor, lung metastases and left hilar lymph node metastasis, underwent surgical resection for left testicular cancer. After orchiectomy and chemotherapy, the patient was successfully treated with wedge resection of the right upper lobe and left upper lobectomy. In conclusion, the current case suggests that some patients with GTS might be successfully treated by surgical resection.
\end{abstract}

Growing teratoma syndrome (GTS) was first advocated by Logothetis in 1982 as a state in which a benign tumor grows after the removal of a primary malignant tumor during or after chemotherapy (1). GTS was defined as the following state: enlargement of metastatic masses during or after systemic chemotherapy for non-seminomatous germ cell tumors (NSGCT); normalization of serum tumor markers; the confirmed diagnosis of mature teratoma in the resected lesions by a pathological examination, without other malignant tumors (2). GTS is a rare phenomenon; the prevalence of the condition in metastatic NSGCT ranges from $1.9 \%$ to $7.6 \%$ (3). GTS is most commonly observed in the retroperitoneum and other organs, such as the inguinal lymph nodes, and the liver has been reported as a site of involvement (4). However, to our knowledge, there are very

Correspondence to: Gouji Toyokawa, MD, Ph.D., Department of Surgery and Science, Graduate School of Medical Sciences, Kyushu University, 3-1-1 Maidashi, Higashi-ku, 812-8582 Fukuoka, Japan. Tel: +81 926425466, Fax: +81 926425482, e-mail: gouji104kawa@gmail.com

Key Words: Growing teratoma syndrome, lung metastases, surgery. few reports on GTS of the lung (5). GTS is resistant to radiotherapy and chemotherapy (2); thus - if applicable - the best treatment for the condition may be complete excision. This is a case report of a lung GTS that was successfully treated by surgical resection and review of the pertinent literature.

\section{Case Report}

A 19-year-old man was referred to his previous doctor for general malaise and nausea. A clinical examination revealed a large left testicular mass. Detailed objective examinations, including chest computed tomography (CT), revealed a left testicular tumor, multiple lung metastases (Figure 1A) and left hilar lymph node metastasis (Figure 1B). He underwent left orchiectomy and a histopathological examination revealed that it was a mixed germ cell tumor. After surgery, he received four cycles of chemotherapy consisting of bleomycin, etoposide, and cisplatin. The treatment led to the regression of the metastatic lesions of the lung as shown by CT (Figure 2A); however, the metastatic left hilar lymph node was observed to have increased in size (Figure 2B). Three cycles of second-line chemotherapy with paclitaxel, isophosphamide, and cisplatin were administered; however, the metastatic left hilar node continued to grow, despite the continued shrinkage of the metastatic lesions in the patient's lung (Figure 2C). Based on the normalization of the patient's serum tumor marker levels ( $\alpha$-fetoprotein, and human chorionic gonadotropin) and the imaging features, GTS was suspected and the patient was referred to our hospital for surgery.

CT revealed one metastatic lesion in the right upper lung, four metastatic lesions in the left upper lung, and one metastatic left hilar lymph node jostling the left pulmonary artery trunk (Figures 3A and B). Thoracoscopic wedge resection of the right upper lung was performed. A pathological examination of the resected lesion revealed that 

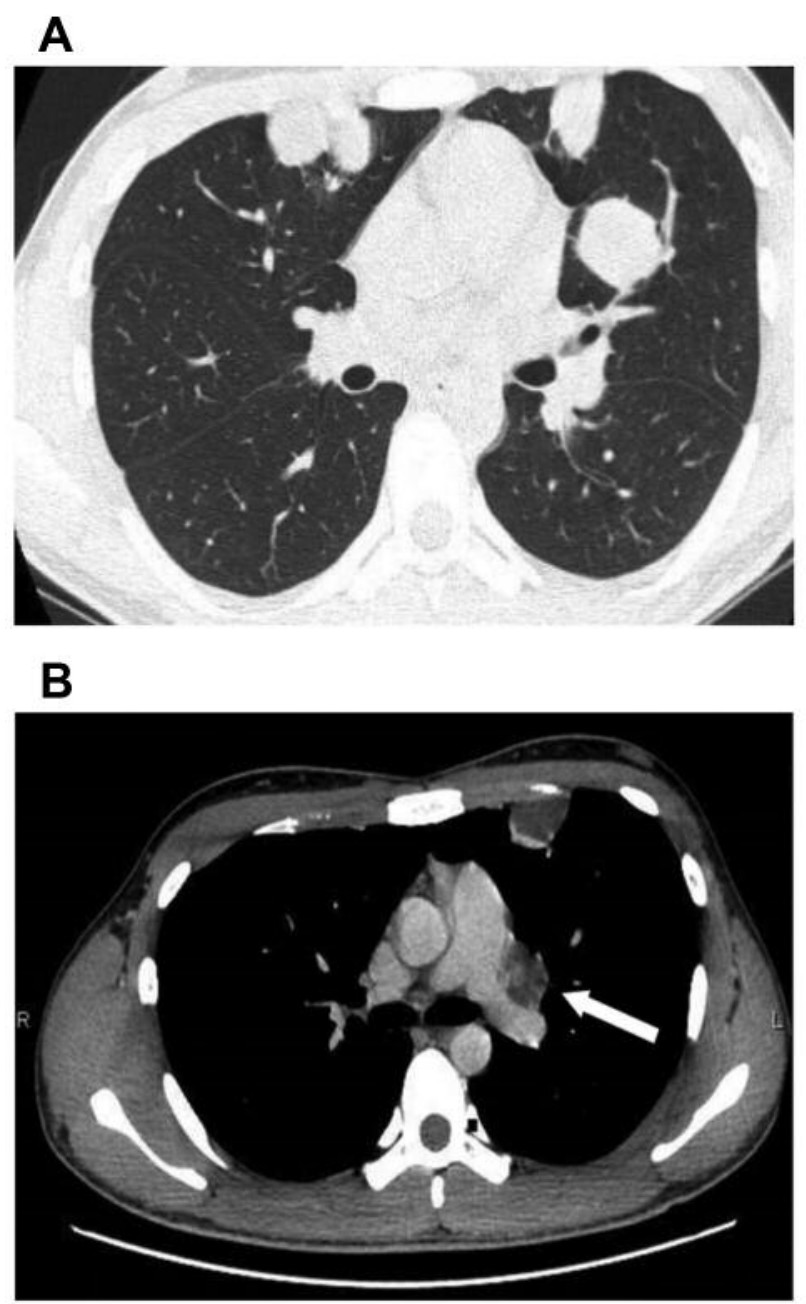

Figure 1. Initial computed tomography imaging of the sites of metastasis. (A) Lung metastases. (B) Left hilar lymph node metastasis (white arrow).

the nodular lesion consisted of necrotic tissue without any evidence of malignant cells or teratoma (Figure 4A). At three weeks after the first surgery, a second operation was performed. Since the intraoperative findings showed no invasion of the left hilar lymph node into the pulmonary artery trunk, the metastatic sites in the lung and lymph node were completely resected by left upper lobectomy and lymph node dissection (Figure 4B).

Pathological findings showed that the nodular lesions were lined by tall columnar epithelial cells without atypia (Figure 4C). Cuboidal cells were seen in the fibrous stroma (Figure 4D). Based on these findings, the tumor was diagnosed as mature teratoma, indicating GTS. At approximately one year after the surgery, he is doing well without any evidence of recurrence.
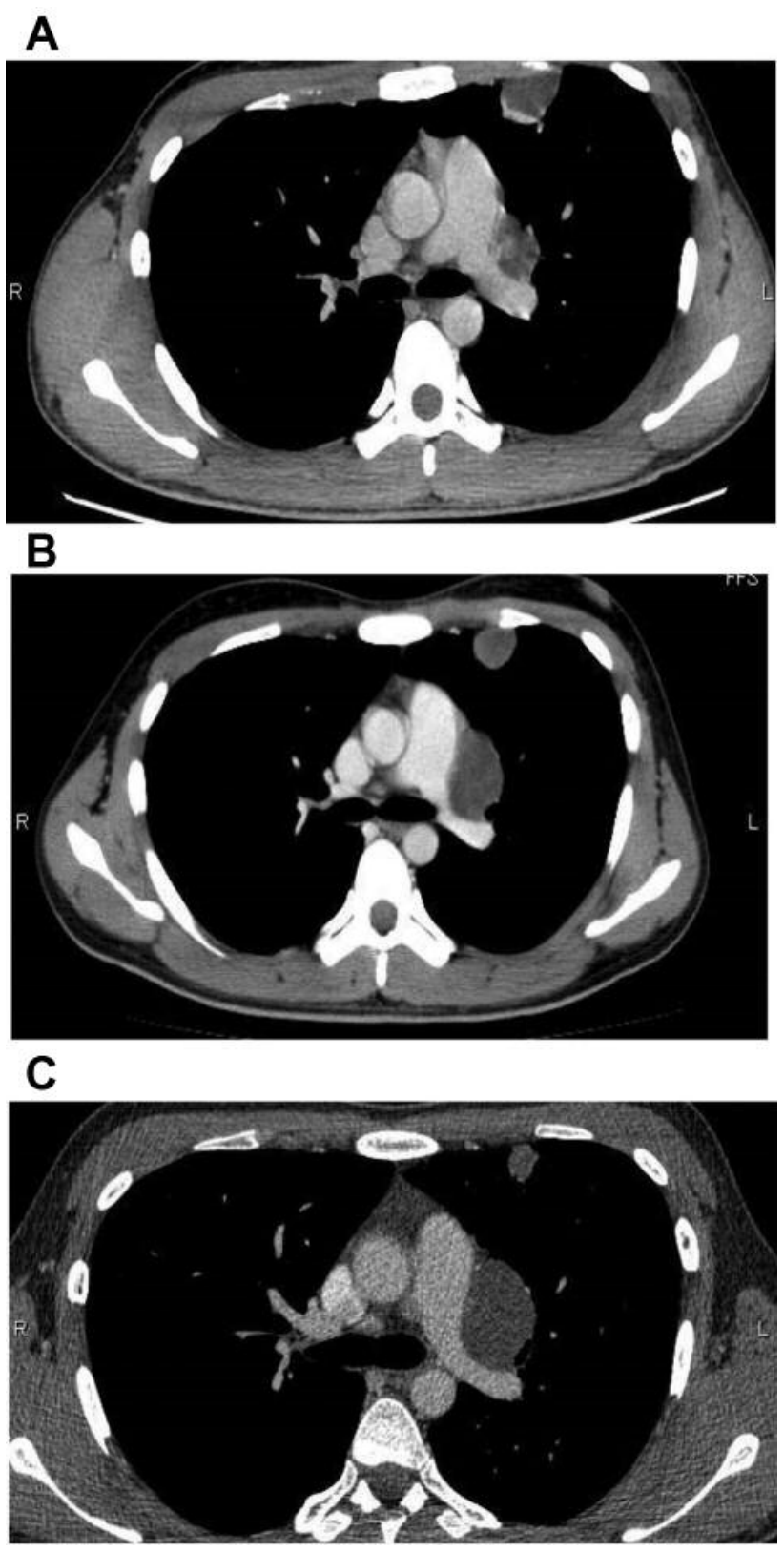

Figure 2. Enlargement of the metastatic left hilar lymph node and the reduction of the volume of one metastatic lesion in the lung during chemotherapy. (A) Before chemotherapy. (B) After first-line chemotherapy (bleomycin, etoposide, and cisplatin: four cycles). (C) After second-line chemotherapy (paclitaxel, isophosphamide, and cisplatin: three cycles).

\section{Discussion}

The concept of GTS was advocated by Logothetis. in 1982 (1); however, the mechanism underlying its development remains to be fully elucidated. Several possible mechanisms have been proposed: first, chemotherapy selects teratoma 
A

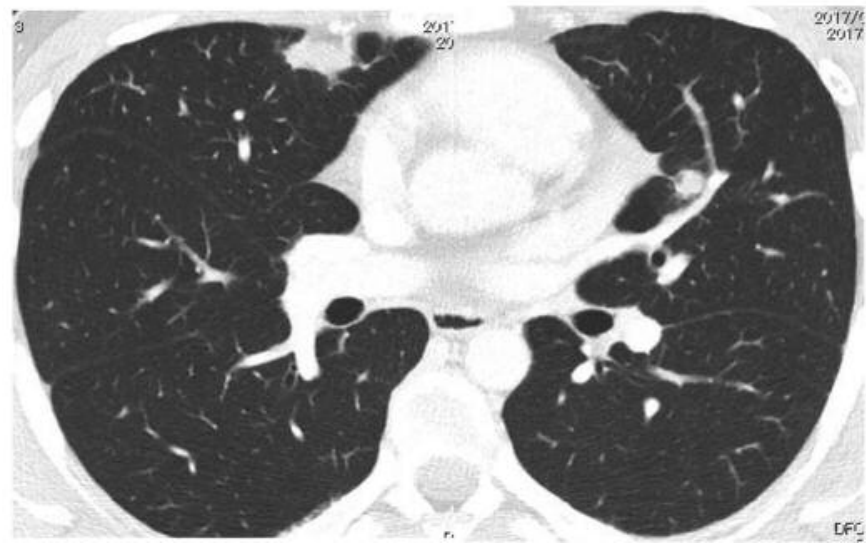

B

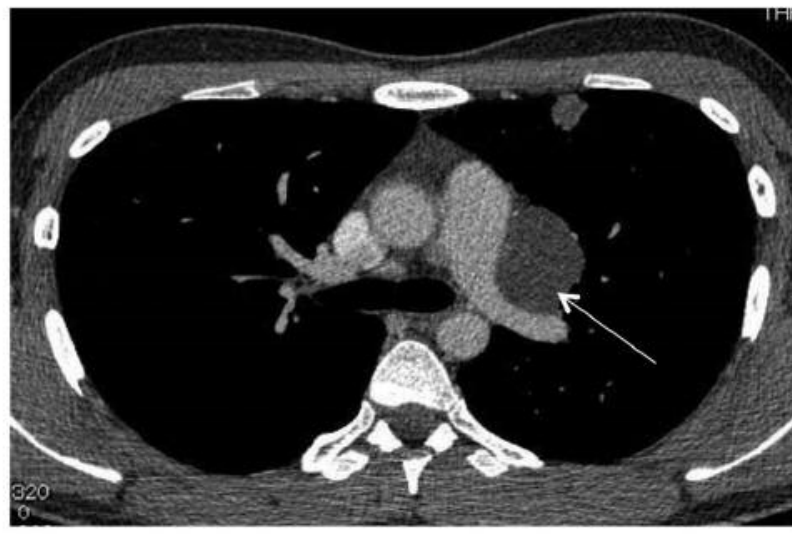

Figure 3. Computed tomography of the metastatic lesions of the lung and the metastatic left hilar lymph node before lung surgery. (A) Lung metastases. (B) Left hilar lymph node metastasis (white arrow).

A

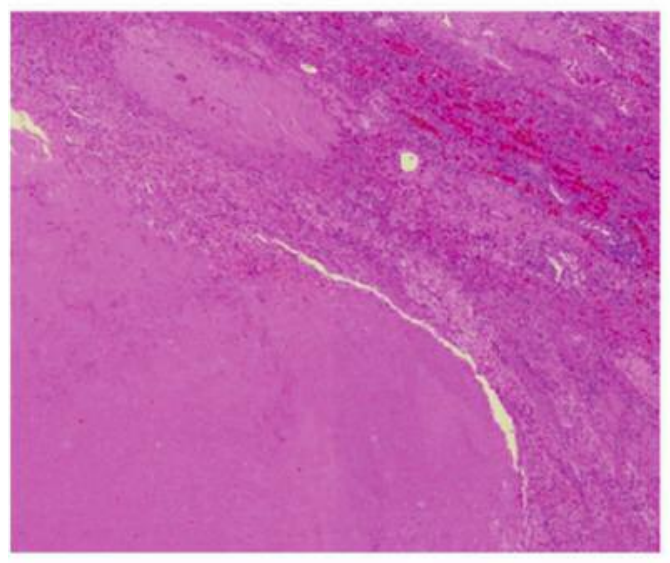

B

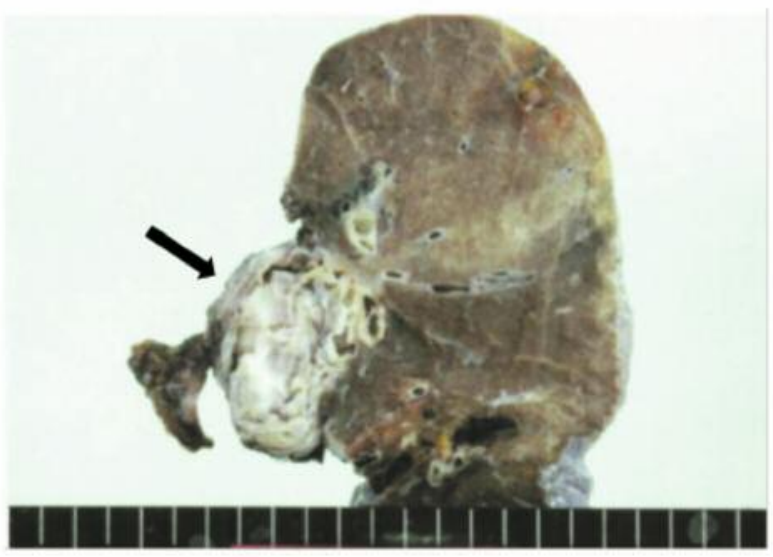

D

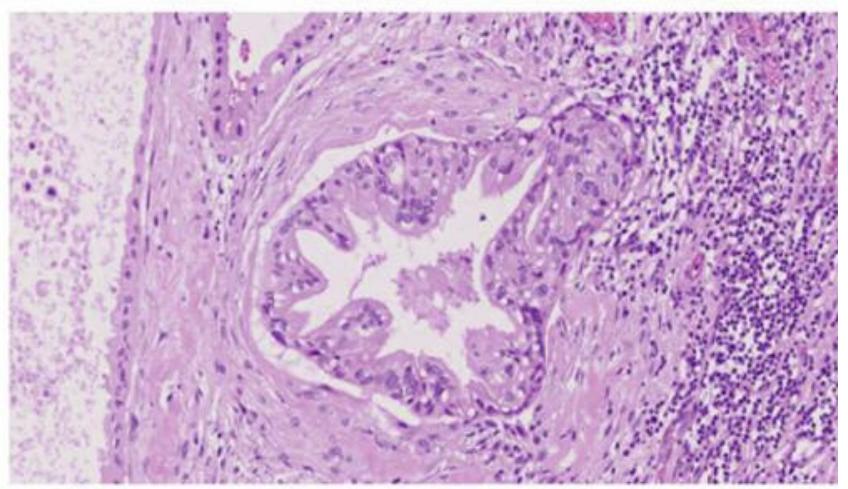

Figure 4. Histopathological findings. (A) Right metastasis: The nodular lesion was full of necrotic tissue. There was no definitive evidence of malignancy or teratoma. (B) Second surgery: macroscopic findings of the resected left upper lobe and the metastatic lymph node (black arrow). (C) Second surgery: nodular lesions are lined by tall columnar epithelial cells without atypia. (D) Second surgery: cuboidal cells are seen in the fibrous stroma. 
cells or induces the transformation of malignant cells into benign cells (6); second, chemotherapy kinetically transforms malignant cells into benign mature teratoma; and third, malignant cells inherently and spontaneously differentiate into benign tissues (7). Since the present patient underwent chemotherapy, these possible mechanisms may explain the pathogenesis of GTS in the current patient.

GTS is a rare phenomenon; its frequency in metastatic NSGCT ranges from $1.9 \%$ to $7.6 \%$ (2). In approximately $90 \%$ of cases, GTS is observed in the retroperitoneum (8). Although other organs, including the mediastinum, supra clavicular lymph nodes, inguinal lymph nodes, forearm, mesentery, and liver, have been reported to be involved, the frequency of their involvement is low (8). Pulmonary GTS is extremely rare, and there are very few reports regarding the complete resection of GTS of the lung. In a report by Yamagishi et al., one and two lesions in the left upper and right lower lungs, respectively, were completely resected by bilateral wedge resection (9). In the current case, left upper lobectomy and wedge resection of the right upper lung was performed for complete resection. Our case suggests that even lobectomy should be considered, if it is oncologically, functionally and technically applicable.

The 5-year overall survival rate of patients with GTS is approximately $90 \%$ (8). In addition, the frequency of recurrence in patients with completely resected GTS is $0-4 \%$, while recurrent GTS is observed in $72-83 \%$ of patients with incomplete resection (10). Furthermore, GTS is resistant to radiotherapy and chemotherapy (2); thus - when applicable the best treatment of GTS may be complete excision. The current patient remains free of recurrence at approximately one year after surgical resection.

\section{Conclusion}

Surgical resection should be considered for patients with GTS if multiple lung metastases can be completely resected.

\section{Conflicts of Interest}

The Authors declare no conflicts of interest in association with the present study.

\section{Acknowledgements}

The Authors thank Brian T. Quinn for his critical comments on the manuscript.

\section{References}

1 Logothetis CJ, Samuels ML, Trindade A and Johnson DE: The growing teratoma syndrome. Cancer 50(8): 1629-1635, 1982.

2 Bentivegna E, Azaïs H, Uzan C, Leary A, Pautier P, Gonthier C, Genestie C, Balleyguier C, Lhomme C, Duvillard P, Morice P and Gouy S: Surgical outcomes after debulking surgery for intraabdominal ovarian growing teratoma syndrome: analysis of 38 cases. Ann Surg Oncol 22(Suppl 3): S964-970, 2015.

3 Jeffery GM, Theaker JM, Lee AH, Blaquiere RM, Smart CJ and Mead GM: The growing teratoma syndrome. Br J Urol 67(2): 195-202, 1991.

4 Boukettaya W, Hochlaf M, Boudagga Z, Ezzairi F, Chabchoub I, Gharbi O, Fatma LB, Sriha B, Mokni M, Kraim CH and Ahmed SB: Growing teratoma syndrome after treatment of a nonseminomatous germ cell tumor: A case report and a review of literature. Urol Case Rep 2(1): 1-3, 2013.

5 Basheda SG, Gephardt G and Meeker DP: The growing teratoma syndrome. Chest 100(1): 259-260, 1991.

6 Priod F, Lorge F, Di Gregorio M, Dupont MV, Nollevaux MC, Faugeras L, Lawson G, Eucher P and D'Hondt L: Recurrent masses after testicular cancer: Growing teratoma syndrome. A case report and review of the literature. Case Rep Oncol 10(3): 910-915, 2017.

7 Hong WK, Wittes RE, Hajdu ST, Cvitkovic E, Whitmore WF and Golbey RB: The evolution of mature teratoma from malignant testicular tumors. Cancer 40(6): 2987-2992, 1977.

8 Maroto P, Tabernero JM, Villavicencio H, Mesía R, Marcuello E, Solé-Balcells FJ, Sola C, Mora J, Algaba F, Pérez C, León X and López López JJ: Growing teratoma syndrome: experience of a single institution. Eur Urol 32(3): 305-309, 1997.

9 Yamagishi T, Shimizu K, Nakata M, Miyaji Y, Nagai A, Kanomata $\mathrm{N}$ and Moriya T: Growing teratoma syndrome of the lung secondary to non-seminomatous germ cell tumor of the testis-a case report. Gan To Kagaku Ryoho 39(10): 1579-1581, 2012.

10 Panda A, Kandasamy D, Sh C and Jana M: Growing teratoma syndrome of ovary: avoiding a misdiagnosis of tumour recurrence. J Clin Diagn Res 8(1): 197-198, 2014. 